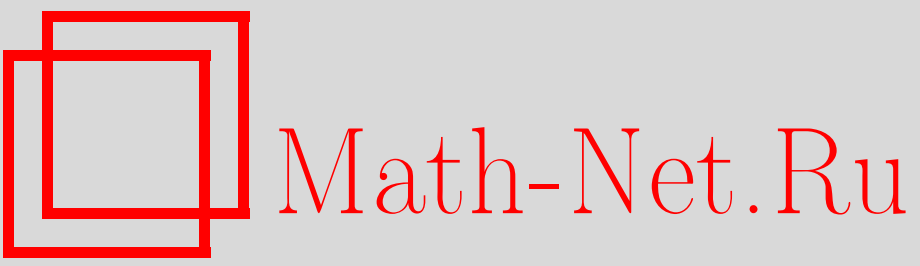

А. Ю. Котов, Заметки о геометрическом квантовании пуассоновых скобок $R$ матричного типа, ТMФ, 1997, том 112, номер 2, 241-248

DOI: https://doi.org/10.4213/tmf1040

Использование Общероссийского математического портала Math-Net.Ru подразумевает, что вы прочитали и согласны с пользовательским соглашением

http://www.mathnet.ru/rus/agreement

Параметры загрузки:

IP : 34.239 .49 .27

26 апреля 2023 г., 07:19:52

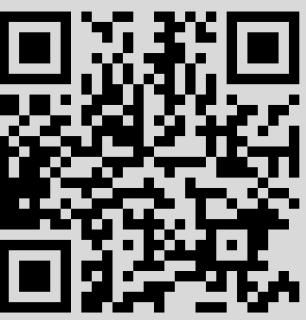




\section{А.Ю. Котов* \\ ЗАМЕТКИ О ГЕОМЕТРИЧЕСКОМ КВАНТОВАНИИ ПУАССОНОВЫХ СКОБОК $R$-МАТРИЧНОГО ТИПА}

Проверяется условие Вайсмана геометрического квантования для пуассоновой пары $R$-матричного типа на коприсоединенной орбите компактной полупростой группы Ли. Показано, что это условие не выполняется для эрмитовых симметрических пространств. Строятся также некоторые примеры пуассоновых пар, для которых условие Вайсмана справедливо.

\section{1. ВВЕДЕНИЕ}

Пусть $G$ - полупростая группа Ли с алгеброй Ли $g$ и $r-R$-матрица Дринфель-

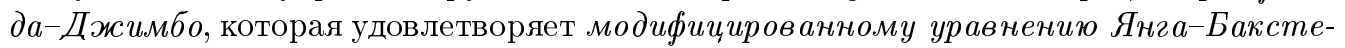
$p a$. Рассмотрим орбиту $\mathcal{O}$ коприсоединенного действия $G$ в $g^{*}$, где $g^{*}-$ дуальное пространство к $g$. На $\mathcal{O}$ сушествует скобка Пуассона $\{,\}_{\text {КкC }}$ (Кириллова-Костанта-Сурьо $-\mathrm{KKC})$.

На $\mathcal{O}$ можно ввести две другие пуассоновы структуры.

1. $G$ действует на $\mathcal{O}$, сохраняя $\mathrm{KKC}$-скобку, поэтому имеется представление

$$
\rho: g \rightarrow \operatorname{Der}\left(C^{\infty}(M),\{,\}_{\mathrm{KKC}}\right) .
$$

Отображение $\{,\}_{r}: C^{\infty}(M)^{\otimes 2} \rightarrow C^{\infty}(M)$, определенное как $\left\{f_{1}, f_{2}\right\}_{r}=\langle(\rho \otimes \rho) r$, $\left.\left(d f_{1} \otimes d f_{2}\right)\right\rangle$, задает кососимметричное умножение на $C^{\infty}(M)$. Оно удовлетворяет тождеству Якоби тогда и только тогда, когда орбита $\mathcal{O} R$-матричного типа. Сужение 3 -вектора $[[r, r]]=\left[r_{12}, r_{13}\right]+\left[r_{12}, r_{23}\right]+\left[r_{13}, r_{23}\right]$, где $r_{12}=r \otimes 1$, на $\mathcal{O}$ в этом случае есть тождественный нуль. Это верно только для орбит $\mathcal{O}$ полупростых элементов, являюшихся симметрическими пространствами, либо для орбит нильпотентных элементов

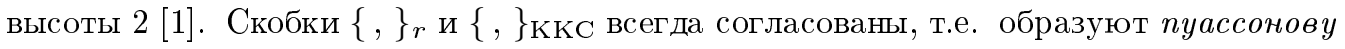
пару на этой орбите.

2. Пусть $K$ - компактная вешественная форма полупростой комплексной группы Ли $G$ и $\mathcal{O}$ - орбита в $k^{*}$. Рассмотрим $\mathcal{O}$ как пуассоново однородное пространство группы Пуассона-Ли $G$ с кограничными скобками Дринфельда-Склянина (ДС), определенными на $G$ следуюшим образом:

$$
\left\{f_{1}, f_{2}\right\}=\left\{f_{1}, f_{2}\right\}_{L}-\left\{f_{1}, f_{2}\right\}_{R}=\left\langle\left(\rho_{L}^{\otimes 2}-\rho_{R}^{\otimes 2}\right)(r), d f_{1} \otimes d f_{2}\right\rangle,
$$

где $\rho_{R}\left(\rho_{L}\right)$ - представление соответствуюшей алгебры Ли $g$ в пространстве $C^{\infty}(M)$ лево- (право-)инвариантными векторными полями.

* Московский государственный университет, Москва, Россия. E-mail: kotov@vitep5.itep.ru 
Собственная структура на $\mathcal{O}$ может быть получена при помощи пуассоновой редукции [2]. Ее также называют скобками ДС и обозначают $\{$,$\} дс. Скобки ККС и ДС$ образуют пуассонову пару тогда и только тогда, когда $\mathcal{O}$ - эрмитово симметрическое пространство [3].

Можно показать, что пуассоновы пары $a\{,\}_{\mathrm{KKC}}+b\{,\}_{\text {дс и }} a\{,\}_{\mathrm{KKC}}+b\{,\}_{r}$ совпадают на эрмитовых симметрических пространствах.

Простой анализ пуассоновых структур, ассоциированных с $r$, показывает, что они, вообще говоря, вырождены. Недавно Вайсман предложил естественное обобшение процедуры геометрического квантования на случай пуассоновых многообразий. Хотя $r$-матричные структуры вводятся в контексте деформационного квантования как квазиклассический предел квантово-групповых структур, представляется интересным проверить условия Вайсмана и в этом случае.

Наша задача - проверить условия Вайсмана геометричекого квантования для скобок

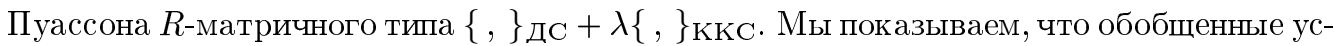
ловия квантования не выполняются в случае эрмитовых симметрических пространств, а также приводим два простых примера, когда это условие справедливо.

\section{2. СКОБКИ ДРИНФЕЛЬДА-СКЛЯНИНА НА ЭРМИТОВЫХ СИММЕТРИЧЕСКИХ ПРОСТРАНСТВАХ}

ОПРЕДЕЛЕНИЕ 1. Группа Ли $G$ называется группой Пуассона-Ли, если она является пуассоновым многообразием, а умножение $G \times G \rightarrow G$ задает морфизм пуассоновых многообразий, где $G \times G$ наделяется прямым произведением пуассоновых структур.

Каждая структура Пуассона-Ли на связной полупростой или компактной группе может быть записана в кограничной форме

$$
\pi(g)=l_{g *}(r)-r_{g *}(r)
$$

где $r \in \bigwedge^{\otimes 2} g$ удовлетворяет модифицированному уравнению Янга-Бакстера, т.е. тензор $[[r, r]]$ инвариантен [4] относительно присоединенного действия $G$.

ОПредЕЛЕниЕ 2. Подгруппа Ли $H$ группы Пуассона-Ли $G$, наделенная собственной структурой Пуассона-Ли, называется подгруппой Пуассона-Ли, если вложение $i: H \hookrightarrow G$ является пуассоновым морфизмом.

Однородное пространство $G / H$ с собственной пуассоновой структурой называется пуассоновым однородным пространством, если естественная проекция $G \rightarrow G / H$ пуассонова.

Предположим, что $H$ связна. Тогда на $G / H$ существует структура однородного пространства Пуассона-Ли, если подпространство $C^{\infty}(G / H) H$-инвариатных функций на $G$ образует подалгебру в пуассоновой алгебре $C^{\infty}(G)[2]$.

Дифференцирование пуассонова тензора $\pi$

$$
d_{e}: g \rightarrow g \wedge g
$$

определенное как $X \rightarrow\left(L_{\bar{X}} \pi\right)(e)$, где $\bar{X}$ - любое векторное поле на $G$, причем $\bar{X}(e)=X$, задает структуру алгебры Ли $[,]^{*}: \bigwedge^{\otimes 2} g^{*} \rightarrow g^{*}$ на $g^{*}$, дуальную $d_{e}=\delta$, и отсюда структуру биалгебры Ли на $g \oplus g^{*}$. 
ПРЕДЛОЖЕНИЕ 1 [2]. Н является подгруппой Пуассона-Ли тогда и только тогда, когда $h^{\perp}-$ идеал в $g^{*}$ (где $h$ - алгебра Ли $\left.H\right)$. $G / H$ есть пуассоново однородное пространство (или однородное пространство Пуассона-Ли) тогда и только тогда, когда $h^{\perp}-$ подалгебра.

Пусть $g$ - полупростая алгебра Ли над $\mathbf{C}, g_{0}-$ та же алгебра, рассмотренная над $\mathbf{R}$.

Пусть $k$ - компактная вешественная форма $g$, т.е. $k$ - множество неподвижных точек обычной антиинволюции Шевалле $\sigma$ алгебры $g$ :

$$
\sigma\left(E_{ \pm \alpha}\right)=-E_{\mp \alpha}, \quad \sigma\left(H_{\alpha}\right)=-H_{\alpha}, \quad \sigma(\lambda X)=\bar{\lambda} \sigma(X),
$$

где $E_{ \pm \alpha}, H_{\alpha}$ - базис Шевалле $g$, построенный по подалгебре Картана $h$.

Пусть $h_{c}$ - подалгебра Картана $k$ и $h=\mathbf{C} h_{c}$. Если выбрать систему $\Delta_{+}$положительных корней $h$ в $g$, то получается разложение

$$
g_{0}=k+a+n_{+},
$$

где $n_{+}=\sum_{\alpha \in \Delta_{+}} g_{\alpha}, a=i h_{c}-$ некомпактная часть подалгебры Картана. Это аддитивное разложение приводит к разложению Ивасавы $K \times A \times N \rightarrow G_{0}$.

$\mathrm{C}$ помощью разложения Ивасавы структура биалгебры Ли на комплексной полупростой алгебре Ли $g$ вводится следуюшим образом:

$$
\delta(X)=[X \otimes 1+1 \otimes X, r],
$$

где $r-R$-матрица Дринфельда-Джимбо:

$$
r=\frac{i}{2} \sum_{\alpha \in \Delta_{+}} E_{\alpha} \wedge E_{-\alpha}
$$

В компактном случае структура биалгебры Ли для $k$ является вешественной формой стандартной структуры биалгебры Ли для $g$, представленной при помоши $r$-матришы:

$$
r_{0}=\frac{1}{4} \sum_{\alpha \in \Delta+} V_{\alpha} \wedge W_{\alpha}
$$

Пусть $P$ - параболическая подгруппа в $G$. Каждая коприсоединенная орбита компактной группы $K$ для произвольной точки $x$ естественно изоморфна расслоению $K / K_{p}$, где $K_{p}=P \cap K$.

Подгруппа $K_{p}$ является подгруппой Пуассона группы Пуассона-Ли $K$. Поэтому каждая коприсоединенная орбита $\mathcal{O}$ в $K$ имеет естественную пуассонову структуру $\pi_{\text {ДС }}$, определенную пуассоновой редукцией. Симплектические листы этой структуры являются $B$-орбитами в $G / P \simeq K / K_{p}$ или клетками Брюа-Шуберта [5].

ОПРЕДЕЛЕнИЕ 3. Две пуассоновы структуры называются совместимыми, если любая их линейная комбинация есть снова пуассонова структура. Это означает, что скобка Схоутена-Нийенхейса соответствуюших бивекторных полей равна нулю.

Пусть $K$ - компактная вешественная форма простой комплексной группы $G$ и $P$ - параболическая подгруппа в $G, K_{p}=P \cap K, p$ и $k_{p}$ - алгебры Ли $P$ и $K_{p}$. Обозначим через $\Delta_{p}$ следующее подмножество положительных корней:

$$
\Delta_{p}=\left\{\alpha \in \Delta_{+} \mid E_{-\alpha} \notin p\right\} .
$$

Полезно заметить, что в случае симметрического разложения

$$
k=k_{x} \oplus m_{x}
$$

только один простой корень $\alpha$ лежит в $\Delta_{p}[6]$.

Пусть $\mathcal{O}_{x} \simeq K / K_{p}$ - коприсоединенная орбита в $k$ фиксированной точки $x$. 
Teоремa 1 [3]. Скобка $K K C\{,\}_{\mathrm{KKC}}$ на $\mathcal{O}_{x}$ совместна со структурой Пуассо-

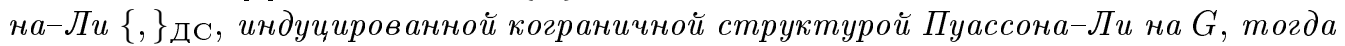
и только тогда, когда орбита является әрмитовым симметрическим пространством.

ПРЕДЛОЖЕНИЕ 2. Структура $\pi_{\lambda}=\pi_{\text {ДС }}+\lambda \pi_{\mathrm{KКС}}$ на эрмитовом симметрическом пространстве вырождается в том и только том случае, если $\lambda \in[-2,0]$.

ДоКАЗАТЕЛЬСТВо ПРЕДЛОЖЕНИЯ 2. Дополним ортонормированную систему

$$
\left\{V_{\alpha}, W_{\alpha} \mid \alpha \in \Delta_{p}\right\} \cap\left\{V_{\alpha}, W_{\alpha} \mid \alpha \in \Delta_{+} \backslash \Delta_{p}\right\}
$$

до ортонормированного базиса для скалярного произведения $-\frac{1}{2} \operatorname{tr}($, ). Коприсоединенное действие $K$ сохраняет форму Картана-Киллинга $\operatorname{tr}($, , ) на $k$, а значит, можно отождествить $\operatorname{Ad} g^{-1}=(\operatorname{Ad} g)^{*}=(\operatorname{Ad} g)^{t}$.

Обозначим матрицы, соответствуюшие бивекторам $r_{0}, r_{p}$ и $r_{\lambda}$, где $r_{\lambda}=l_{g_{*}^{-1}} \pi_{\lambda}(g)=$ $r_{0}-\operatorname{Ad} g^{-1} r_{0}+\lambda r_{p}$, как $\mathbf{R}_{0}, \mathbf{R}_{p}, \mathbf{R}_{\lambda}$.

Если $\operatorname{dim} \mathcal{O}_{x}=2 m$, то

$$
\mathbf{R}_{p}=\frac{1}{4}\left(\begin{array}{ccc}
J_{2 m} & 0 & \ldots \\
0 & 0 & \ldots \\
\vdots & \vdots & \ddots
\end{array}\right),
$$

где

$$
\mathbf{J}_{2 m}=\left(\begin{array}{ccc}
0 & 1 & \ldots \\
-1 & 0 & \ldots \\
\vdots & \vdots & \ddots
\end{array}\right) .
$$

Нетрудно видеть, что структура $\pi_{\lambda}(g)$ вырождается тогда и только тогда, когда вырождается левый верхний $2 m \times 2 m$-минор матрицы $\mathbf{R}_{\lambda}$. Ранг левого верхнего $2 m \times$ $2 m$-минора равен рангу матрицы $16 \mathbf{R}_{\lambda} \mathbf{R}_{p}$. Очевидно, что

$$
\operatorname{det}\left(16 \operatorname{Ad} g^{t} \mathbf{R}_{0} \operatorname{Ad} g \mathbf{R}_{p}+\left(\lambda+1 \mathbf{E}_{p}\right)\right)=0,
$$

где $\mathbf{E}_{p}=-16 \mathbf{R}_{0} \mathbf{R}_{p}=-16 \mathbf{R}_{p}^{2}$.

Также можно показать, что

$$
\operatorname{det}\left(16 \operatorname{Ad} g^{t} \mathbf{R}_{0} \operatorname{Ad} g \mathbf{R}_{p}+(\lambda+1) \mathbf{E}\right)=0 .
$$

Это характеристическое уравнение для матрицы $16 \mathrm{Ad} g^{t} \mathbf{R}_{0} \operatorname{Ad} \mathbf{R}_{p}$. Следовательно,

$$
|\lambda+1| \leq\left\|16 \operatorname{Ad} g^{t} \mathbf{R}_{0} \operatorname{Ad} g \mathbf{R}_{p}\right\| \leq 1,
$$

где $\|\mathbf{A}\|=\operatorname{supp}_{\|v\|=1}\langle\mathbf{A} v, v\rangle$.

Докажем необходимое условие.

Рассмотрим подгруппу $K_{\alpha} \subset K$ с подалгеброй Ли $k_{\alpha}=\mathbf{R} i H_{\alpha} \oplus \mathbf{R} V_{\alpha} \oplus \mathbf{R} W_{\alpha}$, где $\alpha$ - единственный корень из $\Delta_{p}$. Хорошо известно, что такая глобальная подгруппа сушествует.

Орбита $K_{\alpha}$-действия на $\mathcal{O}_{x}$ естественно изоморфна обычной сфере. Более того, симплектические листы собственной структуры на "малой" орбите совпадают с пересечением симплектических листов на $\mathcal{O}_{p}$ и этой орбитой (если рассмотреть ее как пуассоново однородное пространство подгруппы Пуассона-Ли $\left.K_{\alpha}\right)$. Скобка $\pi_{\lambda}$ на $C P^{1}$ вырождается для всех $\lambda \in[-2,0]$. Отсюда делается вывод, что структура $\pi_{\lambda}$ является вырожденной для всех $\lambda \in[-2,0]$.

Пусть $w$ - элемент из нормализатора $N(H)$, имеюший максимальную длину в группе Вейля $W\left(\Delta_{+}\right)$. Тогда $\operatorname{Ad} w\left(r_{0}\right)=-r_{0}$, т.к. $\operatorname{Ad} w_{\mid h}: \Delta_{+} \rightarrow-\Delta_{+}$. 
ПРЕДЛОЖЕНИЕ $3 . l_{w^{*}} \pi_{\lambda}(g)=-\pi_{-(\lambda+2)}(g)$.

ДОКАЗАТЕЛЬСТВО.

$$
\begin{aligned}
\left(l_{w^{*}}\right) \pi_{\lambda}(g) & =l_{g^{*}}\left(\pi_{\lambda}\left(w^{-1} g\right)\right)=l_{g^{*}} l_{\left(w^{-1} g\right)^{*}}^{-1} \pi_{\lambda}\left(w^{-1} g\right)= \\
& =l_{g^{*}}\left(r_{0}-\operatorname{Ad} g^{-1}\left(w^{-1} g\right) r_{0}+\lambda r_{p}\right)=l_{g^{*}}\left(-r_{0}+\operatorname{Ad} g^{-1} r_{0}+(\lambda+2) r_{p}\right)= \\
& =-l_{g^{*}}\left(r_{0}-\operatorname{Ad} g^{-1} r_{0}-(\lambda+2) r_{p}\right)=-\pi_{-(\lambda+2)}(g) .
\end{aligned}
$$

Таким образом, топологические свойства (т.е. топологическая структура симплектических листов, условия квантования) структур $\pi_{\lambda}$ и $\pi_{-(\lambda+2)}$ эквивалентны. Если $\lambda=0$, то $\pi_{0}=\pi_{\text {ДС эквивалентно } \pi_{-2} .}$

\section{3. ГЕОМЕТРИЧЕСКОЕ КВАНТОВАНИЕ СКОБОК ПУАССОНА $R$-МАТРИЧНОГО ТИПА}

ОПРЕДЕЛЕнИЕ 4. Геометрическим квантованием пуассоновой структуры называется отображение из подмножества гладких функций на многообразии $M$ в множество эрмитовых операторов, действующих в пространстве глобальных сечений линейного эрмитова расслоения над $M$. Кроме того, должно удовлетворяться следующее уравнение:

$$
\left\{\widehat{f_{1}, f_{2}}\right\}=\frac{2 \pi i}{\hbar}\left[\widehat{f}_{1}, \widehat{f}_{2}\right]
$$

где $f_{1}, f_{2} \in C^{\infty}(M)$ и $\widehat{f}$ - операторный образ $f, \hbar$ - постоянная Планка.

В симплектическом случае ответ следуюший [7]:

$$
\widehat{f}=f+\frac{\hbar}{2 \pi i} \nabla_{c(f)}
$$

здесь $c(f)$ - векторное поле, действуюшее как $c(f) g=\{f, g\}$ для всех $g \in C^{\infty}(M)$, $\nabla$ - ковариантная производная, задающая линейную связность с формой кривизны $\Omega$, и $\Omega=\frac{1}{\hbar} \omega$, где $\omega$ - симплектическая форма на $M$. Известно, что $\Omega$ - форма кривизны некоторого комплексного линейного расслоения тогда и только тогда, когда класс когомологий $\Omega$ целочисленный. Эрмитова структура в линейном расслоении, которая наделяет пространство сечений структурой гильбертова пространства, существует и ковариантно-постоянна, только если форма $\Omega$ вешественна, т.е. $\omega \in \hbar H^{2}(M, Z)$.

В невырожденном случае некоторые приложения указанной схемы были предложены в $[8]$.

Пусть $M^{m}$ - гладкое многообразие с пуасоновой структурой, определенной бивекторным полем $\pi$. Пусть $\mathcal{A}$ - пуассонова алгебра, где $\mathcal{A}=\left(C^{\infty}(M),\{\},\right)$.

Будем говорить, что $M$ допускает предквантование над комплексным линейным расслоением $L \rightarrow M$, если выполнены следуюшие условия:

$$
\left\{\widehat{f_{1}, f_{2}}\right\}=\frac{2 \pi i}{\hbar}\left[\widehat{f_{1}}, \widehat{f}_{2}\right]
$$

где $\widehat{f}_{j}=f_{j}+\frac{\hbar}{2 \pi i} \nabla f$.

Дифференциальный оператор $\nabla f$ действует в пространстве глобальных сечений как $\nabla_{f} g s=\{f, g\} s+g \nabla_{f} s$ для всех $f, g \in C^{\infty}(M), s \in \Omega^{0}(L)$. 
Рассмотрим оператор $\delta: \Upsilon^{*}(M) \rightarrow \Upsilon^{*+1}(M)$ следуюшего пуассонова дифференцирования:

$$
\delta f=c(f), \quad \delta X=[[\pi, X]],
$$

где [[, ]] - скобка Схоутена-Нийенхейса, $f \in C^{\infty}(M), X \in \Upsilon^{*}(M)$ - поливекторное поле. Легко показать, что

$$
\mathcal{P}(\lambda)\left(\alpha_{1}, \ldots, \alpha_{k}\right)=(-1)^{k} \lambda\left(\mathcal{P}\left(\alpha_{1}\right), \ldots, \mathcal{P}(\alpha)\right) \text { для всех } \alpha_{1}, \ldots, \alpha_{k} \in \Omega^{1}(M),
$$

где $\beta(\mathcal{P}(\alpha))=\pi(\alpha, \beta) \forall \alpha, \beta \in \Omega^{1}(M), \lambda \in \Omega^{k}(M)$ и $\mathcal{P}(\lambda) \in \Upsilon^{k}(M)$. $\mathcal{P}$ - оператор Гамильтона, определенный тензором $\pi$.

Можно также показать, что тождество Якоби для скобки Пуассона $\{$,$\} эквива-$ лентно условию $\delta^{2}=0$, которое удовлетворяется при $[[\pi, \pi]]=0$. Следовательно, $\left(\Upsilon^{*}(M), \delta\right)$ - комплекс, называемый пуассоновым, с пуассоновыми когомологиями $[9]$ :

$$
H^{k}(\mathcal{A})=\frac{\operatorname{Ker}\left\{\delta^{k}: \Upsilon^{k}(M) \rightarrow \Upsilon^{k+1}(M)\right\}}{\operatorname{Im}\left\{\delta^{k-1}: \Upsilon^{k-1}(M) \rightarrow \Upsilon^{k}(M)\right\}}
$$

Теорема 2 [8]. Пуассоново многообразие $(M, \pi)$ допускает квантовое расслоение тогда и только тогда, когда существуют векторное поле $X$ и замкнутая 2-форма $\omega$, представляющая собой целочисленный класс когомологий $M$, такие, что выполняется условие

$$
\pi+L_{X} \pi=\mathcal{P}(\omega) .
$$

В этом случае класс $\frac{i}{2 \pi}[c] \in H^{2}(\mathcal{A})$, который называется классом Пуассона-Черна $c_{1}(\pi)$, корректно определен. Это образ целочисленного класса из $H^{2}(M, Z)$.

ПримеР 1. Пусть $(p, q) \in R^{2}$ - стандартное симплектическое пространство со следуюшим нетривиальным коммутационным соотношением: $\{p, q\}=1$.

Мы вводим новую структуру

$$
\left\{f_{1}, f_{2}\right\}_{\text {new }}=\left\{f_{1}, h_{1}\right\}\left\{f_{2}, h_{2}\right\}-\left\{f_{1}, h_{2}\right\}\left\{f_{2}, h_{1}\right\}, \quad \text { где } h_{1}=p, \quad h_{2}=p q .
$$

Здесь $\pi=p \frac{\partial}{\partial p} \wedge \frac{\partial}{\partial q}$ и $\{p, q\}=p$. Пуассонова структура вырождается при $p=0$. Так как $H^{*}\left(R^{2}\right)=0$, мы можем взять тривиальное комплексное линейное расслоение $R^{2} \times C \rightarrow R^{2}$, где множество глобальных сечений эквивалентно $C^{\infty}(M) \otimes C$. Поэтому $\omega=0$. Наше уравнение $\pi+[[X . \pi]]=0$ имеет нетривиальное решение $X=q \frac{\partial}{\partial q}$. Эта скобка также была проквантована при помощи рядов Дринфельда [10].

ПримеР 2. Рассмотрим орбиту нильпотентного элемента в $g^{*}$, где $g=\operatorname{sl}(2, \mathbf{R})$. Будем отождествлять $g^{*}$ и $g$ при помощи скалярного произведения $\operatorname{tr}\langle\rangle$. Введем координатную систему $\left(x_{1}, x_{2}, x_{3}\right)$ в $g$ так, чтобы каждая матрица записывалась в форме

$$
\left(\begin{array}{cc}
x_{2} & x_{1}+x_{3} \\
x_{1}-x_{3} & -x_{2}
\end{array}\right) .
$$

Уравнение $x_{1}^{2}+x_{2}^{2}=x_{3}^{2}$ соответствует сингулярной орбите нильпотентного элемента. В сфрерических координатах

$$
\begin{aligned}
& x_{1}=r \cos \phi \sin \theta, \\
& x_{2}=r \sin \phi \sin \theta, \\
& x_{3}=r \cos \theta
\end{aligned}
$$


эта орбита соответствует $\theta=\frac{\pi}{4}$.

$R$-матрица Дринфельда-Джимбо на $\bigwedge^{2} g$ определяет $r$-скобки:

$$
\{r, \phi\}_{r}=-\frac{r}{2} \sin \phi
$$

которые вырождаются, если $\sin \phi=0$.

Геометрическое предквантование в тривиальном комплексном линейном расслоении строится следующим образом:

$$
\widehat{F}=F+\frac{\hbar}{2 \pi i} c(F)+X(F),
$$

где $X=-r \ln r \frac{\partial}{\partial r}$ - решение уравнения (21).

Рассмотрим геометрическое квантование семейства скобок Пуассона $R$-матричного типа $\pi_{\lambda}$ на $\mathcal{O}_{x}$-орбите компактной полупростой группы Ли $K$.

В частном случае, если $K=S U(2), \mathcal{O}_{x}=C P^{1}$, то $\pi_{\lambda}$ может быть записано в форме

$$
\pi_{\lambda}=-\frac{i}{2}\left(1+|z|^{2}\right)\left(\lambda+(\lambda+2)|z|^{2}\right) \frac{\partial}{\partial z} \wedge \frac{\partial}{\partial \bar{z}} .
$$

Для $\lambda \in[-2,0]$ пуассонов бивектор вырождается на множестве

$$
\Xi_{\lambda}=\left\{\left.z|| z\right|^{2}=-\frac{\lambda}{\lambda+2}\right\} .
$$

Условие геометрического квантования следуюшее: $\pi_{\lambda}+\delta X=\mathcal{P} \omega$, где $[\omega] \in$ $H^{2}\left(C P^{1}, Z\right)$. Это условие эквивалентно тому, что $\mathcal{P}^{-1}\left(\pi_{\lambda}\right)+d\left(\mathcal{P}^{-1} X\right)=\omega$ везде, кроме $z \in \Xi_{\lambda}$. Другими словами,

$$
-\frac{2}{i} \frac{1}{1+|z|^{2}} \frac{1}{\lambda+(\lambda+2)|z|^{2}} d z \wedge d \bar{z}=\omega-d\left(\frac{1}{\lambda+(\lambda+2)|z|^{2}} \sigma\right),
$$

где $\sigma \in \Omega^{1}\left(C P^{1}\right)$.

Используя формулу Стокса, вычисляем

$$
\begin{aligned}
\iint_{|z| \geq \xi} \mathcal{P}^{-1}\left(\pi_{\lambda}\right)= & \iint_{|z| \geq \xi}\left[-d\left(\frac{1}{\lambda+(\lambda+2)|z|^{2}}\right) \sigma+\omega\right]- \\
& -2 \pi \ln \frac{\lambda+(\lambda+2) \xi^{2}}{(\lambda+2)\left(1+\xi^{2}\right)}=\phi(\xi)-\frac{1}{\lambda+(\lambda+2) \xi^{2}} \psi(\xi),
\end{aligned}
$$

где

$$
\phi(\xi)=\iint_{|z| \geq \xi} \omega, \quad \psi(\xi)=\oint_{|z|=\xi} \sigma, \quad \phi, \psi \in C^{\infty}\left(\left[\xi_{0},+\infty\right]\right), \quad \xi_{0}^{2}=-\frac{\lambda}{\lambda+2} .
$$

При $\xi \rightarrow+\xi_{0}$ левая часть уравнения содержит логарифмическую особенность, в то время как правая - полюсную. Поэтому условие геометрического квантования не выполняется. 
ПРЕДЛОЖЕНИЕ 4. Для всех $\lambda \in[-2,0]$ условие геометрического квантования для $\pi_{\lambda}$ не выполняется.

ДокаЗАтЕЛЬСтво. Рассмотрим снова подгруппу $K_{\alpha} \subset K$ с подалгеброй Ли $k_{\alpha}=$ $R i H_{\alpha} \oplus R V_{\alpha} \oplus W_{\alpha}$, где $\alpha \in \Delta_{+}$и $\alpha-$ простой.

Орбита $\mathcal{O}_{x}$ действия $K_{\alpha}$ является пуассоновым подмногообразием относительно

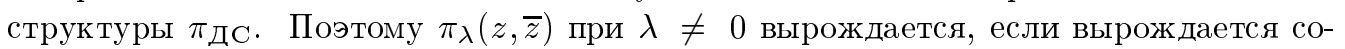
ответствуюшая структура на "маленькой" орбите с тем же $\lambda$. Отсюда уравнение $\pi_{\lambda}+\delta X=\mathcal{P}(\omega)$ для всех неособых $z \in \mathcal{O}_{\alpha}$ может быть редуцировано к уравнению $\left(\mathcal{P}^{-1} \pi_{\lambda}\right)+d\left(\mathcal{P}_{-1}(X)\right)=\omega$, которое обсуждалось ранее. Это уравнение не имеет решений на $C P^{1}$, что может быть показано путем прямых вычислений. Если $\lambda=0$, то

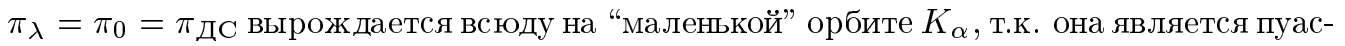
соновым подмногообразием. Однако $l_{w} * \pi_{\lambda}=-\pi_{-(\lambda+2)}$, отсюда следует, что для $\lambda=0$ структура не допускает геометрического квантования.

Автор выражает признательность В. Рубцову и Б. Стернину за постановку задачи и полезные обсуждения.

\section{Список литературы}

[1] D. Gurevich, D. Panyushev. Duke Math. J. 1994. V. 73. № 2. P. 249-255.

[2] M. Semenov-Tyan-Shansky. Publ. RIMS, Kyoto Univ. 1985. V. 21. P. 1237-1260.

[3] S. Khoroshkin, A. Radul, V. Rubtsov. Commun. Math. Phys. 1993. V. 152. P. 299-315.

[4] V. Drinfeld. Quantum groups. In: Proc. ICM, Berkeley. V. 1. Am. Math. Soc., 1986. P. 789-920.

[5] J. H. Lu, A. Weinstein. J. Differ. Geom. 1990. V. 31. P. 501-526.

[6] S. Helgason. Differential geometry, Lie groups and symmetric spaces. New York: Academic Press, 1978.

[7] А. А. Кириллов. Элементы теории представлений. М.: Наука, 1972.

[8] I. Vaisman. J. Math. Phys. 1991. V. 32. № 12. P. 3339-3345.

[9] A. Lichnerovicz. J. Differ. Geom. 1977. V. 12. P. 253-300.

[10] D. Gurevich, V. Rubtsov, N. Zobin. J. Geom. Phys. 1992. V. 9. № 1. P. 25-44.

Поступила в редакцию 25.XII.1996 г.

\section{A. Yu. Kotov \\ REMARKS ON GEOMETRIC QUANTIZATION OF $R$-MATRIX TYPE POISSON BRACKETS}

We check the Vaisman condition of geometric quantization for $R$-matrix type Poisson pencil on a coadjoint orbit of a compact semisimple Lie group. It is shown that this condition is not satisfied for hermitian symmetric spaces. We also construct some examples when the Vaisman condition takes place. 